\title{
Surface smoothening of the inherent roughness of micro-lenses fabricated with 2-photon lithography
}

\author{
Helmut Schift, ${ }^{1, *}$ Robert Kirchner, ${ }^{2}$ Nachiappan Chidambaram, ${ }^{1}$ Mirco Altana ${ }^{3}$ \\ ${ }^{1}$ Paul Scherrer Institute, Laboratory for Micro and Nanotechnology, 5232 Villigen PSI, Switzerland \\ 2 Technische Universität Dresden, IHM, 01062 Dresden, Germany \\ ${ }^{3}$ Heptagon Oy, Moosstrasse 2, 8803 Rueschlikon, Switzerland
}

\begin{abstract}
Two-photon polymerization by direct laser writing enables to write refractive micro-optical elements with sub- $\mu \mathrm{m}$ precision. The trajectories and layering during the direct writing process often result in roughness in the range of the writing increment, which has adverse effects for optical applications. Instead of increasing overlap between adjacent voxels, roughness in the range of $100 \mathrm{~nm}$ can be smoothed out by post-processing. For this a method known as TASTE was developed, which allows polishing of surfaces without changing the structural details or the overall shape. It works particularly well with thermoplastic polymers and enables sub-10 nm roughness. The optical quality was confirmed for an array with several 100 microlenses.
\end{abstract}

Keywords: Micro-lens array, glass transition temperature, surface roughness, polymer, 2-photon polymerization

\section{INTRODUCTION}

This work is a short review of a technology, which enables to fabricate microlens structures with ultra-smooth, optical grade surfaces. It is particularly suitable for refractive lenses made by novel 3D lithographic methods, where surface roughness is constituted by undulations stemming from the layer-to-layer trajectory based writing [1-3]. Instead of improving surface roughness by applying high overlap of trajectories or time-consuming writing strategies, we chose a post-processing method based on selective thermal reflow. The traditional reflow process, well established for manufacturing refractive lenses, uses surface energy minimization in small pads of molten polymer (resist) features, which in their viscous state obtain the optimum convex shape given by the boundaries of the initial resist volume and a super-smooth surface [4]. With only local modification of the polymer's ability to flow, it can be applied to a range of other shapes, made by 3D lithography. The reflow process becomes a contact-less polishing process, because it only levels out surface undulations without removal of material. It is a self-optimizing rearrangement by material displacement between microscopic mounds and valleys while at the same time preserving the (geometrical) envelope of the original lens shape. It is highly desired in a world of 3D lithography where amazing continuous and stepped topographies can be achieved, however, with roughness not tolerable for optical applications due to the 3D nature of the writing process. The roughness needed for such applications is typically below $5 \mathrm{~nm}$ for the visible range, i.e., about $1 / 100^{\text {th }}$ of the wavelength used, which results in low stray light, high resolution and high imaging contrast $[5,6]$. The following publications serve as references for the proposed process and its underlying physical principles [7-9].

In this introduction, we will highlight two aspects of the surface smoothening: The origin of the roughness stemming from two-photon polymerization (2PP) by direct laser writing (DLW), and the surface selective TASTE process (thermally activated selective topography equilibration). The latter is well described in $[10,11]$ and was initially applied to electron beam lithography (EBL) to generate smooth linear slopes or convex and concave shapes from stepped resist profiles. The roughness is inherent to all DLW schemes and particularly to those using $2 \mathrm{PP}$ where material is polymerized within a small volume element (voxel). The confinement of the structure formation to the writing trajectory results in a pronounced roughness, which is typically larger than the roughness by material and chemical process related inhomogeneity. It also adds to other machine-related defects such as sub-field stitching but often is regular in nature.

* helmut.schift@psi.ch; phone +41 56310 2839; fax +41 56310 2646; www.psi.ch/lmn 


\section{LAYERING VERSUS POSTPROCESSING FOR ROUGHNESS SMOOTHENING}

There are novel fabrication schemes including 3D lithography methods such as grayscale lithography and 2PP lithography that build for example micro-optical lenses out of thin layers. Grayscale approaches are either based on masks with varying transmission grades (grayscale masks) or direct writing methods [12,13]. While the first one is well established for i-line lithography $(365 \mathrm{~nm}$ ), higher resolution methods are based on sequential and scanned photon, electron or ion beam writing. DLW with a lateral resolution of about $700 \mathrm{~nm}$ is well suited for refractive micro-optics, its vertical limitation to resist films below $30 \mu \mathrm{m}$ limits its use for freeform and complex designs beyond this thickness. This is mainly due to the fact that the entire film is exposed along the beam path when using single photon absorption processes. Similarly, the higher resolution grayscale methods based on electron and ion beams are mostly suitable for diffractive optics with film resist thickness below $10 \mu \mathrm{m}$ for electrons and even thinner for ions [14,15]. A true 3D capable method such as 2PP-DLW allows for full freeform designs without restrictions on the size of the micro-optics [16]. Exemplary realized designs are micro-prisms [2], single-tier micro-lenses [17,18], multi-tier lenses with undercuts and hidden features [19]. As mentioned above, the typical limitation of 2PP-DLW is the writing time and thus writing area and height. This is not only because of the higher resolution of the beam in lateral direction, but also due to the additional confinement of the polymerizing laser beam in vertical direction, which makes fully 3D structures possible but also require intensive layering for more bulky structures. The laser beam focus (a spheroid called voxel), is scanned through a viscous material and polymerizes the material on its track, which means that the structure is built from trajectories being stacked like woodpiles.

There are three main strategies to deal with the roughness concern in 2PP (Figure 1): (a) voxel: increasing the voxel overlap by having a finer vertical/horizontal discretization or reducing the voxel size by varying the polymerization threshold/dose, (b) writing strategies: varying the vertical discretization based on the curvature of the feature to be written or writing curved out-of-plane instead of in-plane trajectories, (c) post processing: the feature is transferred into a thermoplastic material and controlled reflow is carried out. In the first strategy, increasing overlap reduces the roughness to a certain extent [20], but resist tends toward micro-explosions for an overlap being too high. Micro-explosions are caused by high laser dose or high voxel overlap due to the dielectric breakdown of the exposed and polymerized material by an electron avalanche mechanism [21]. Reducing the voxel size has only small process latitude. In addition, most users in 2PP-DLW already work with the lowest voxel size possible. With variable vertical discretization, roughness can be reduced [22], but one needs to dynamically tune the dose to avoid micro-explosions. Out-of-plane trajectories can give some improvements [23] but this is essentially shifting the roughness problem from ' $z$ ' axis to ' $x$ or $y$ ' axis. Furthermore, high resolution features (tips of concave refractive structures) and high aspect ratio structures, will suffer the most from writing strategy approaches that will remain reserved for special geometries [19] that do not allow for using reflow at the moment. We believe a universal post processing is the appropriate strategy for attaining a super smooth surface while having limited change in the surface profile alongside acceptable writing times.

\section{Voxel size}

- Laser dose / threshold

- Exposure optics and resist with «diffusive» component

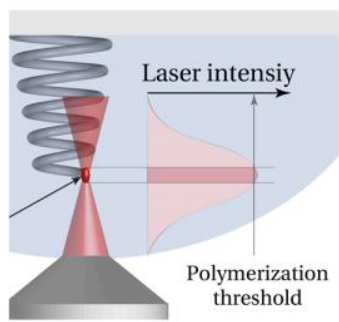

\section{Writing strategies}

- Increasing the voxel overlap

- Variable height slicing

- Arc scanning

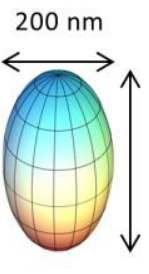

\section{Post processing}

- «Reflow» of resist

- Replication required
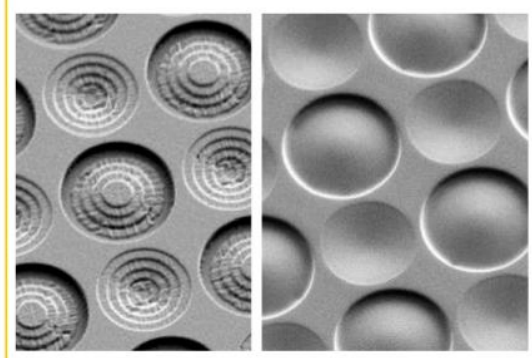

Figure 1. Alternatives for surface smoothening by variation of voxel size, writing strategies and post processing. 


\begin{tabular}{|c|c|c|c|c|}
\hline Origin of roughness & $\begin{array}{l}\text { Writing increment } \\
\text { (lateral/vertical) }\end{array}$ & Stitching (lateral) & $\begin{array}{l}\text { Material } \\
\text { inhomogeneity }\end{array}$ & Laser power variation \\
\hline Nature & $\begin{array}{l}\text { Regular, design } \\
\text { dependent }\end{array}$ & $\begin{array}{l}\text { Writing-field } \\
\text { stitching, geometric } \\
\text { distortion of } \\
\text { individual writing } \\
\text { fields (barrel / } \\
\text { cushion), anisotropic } \\
\text { shrinkage, writing } \\
\text { direction }\end{array}$ & $\begin{array}{l}\text { Incomplete } \\
\text { polymerization, } \\
\text { swelling, scattering at } \\
\text { refractive index } \\
\text { variations, impurities, } \\
\text { already written } \\
\text { structures or at } \\
\text { interfaces }\end{array}$ & $\begin{array}{l}\text { Statistics, over- } \\
\text { exposure and resist } \\
\text { "explosion" }\end{array}$ \\
\hline \multirow[t]{2}{*}{ Strategy } & $\begin{array}{l}\text { Increase of voxel size } \\
\text { (microscope lens, } \\
\text { exposure parameters, } \\
\text { diffusive resist) }\end{array}$ & $\begin{array}{l}\text { Stitching matched to } \\
\text { sub-element size, } \\
\text { overlap, alternation } \\
\text { of writing direction }\end{array}$ & Process control & $\begin{array}{l}\text { Short writing times, } \\
\text { process control }\end{array}$ \\
\hline & Higher overlap & & & \\
\hline
\end{tabular}

Table 1. Different origins of roughness and strategies to remove them during the 2PP-DLW process.

While piezo drives of current 2PP-DLW systems allow for the scanning of trajectories in all directions, fast writing is often achieved when the scanning mechanism in lateral direction is done via fast galvanic scanners. This allows layer-bylayer writing for which particularly the layering in vertical direction becomes the limiting factor. In dependence of the final 3D design and the finite focal spot size between some $200 \mathrm{~nm}$ in lateral up to $4^{\prime} 000 \mathrm{~nm}$ in vertical direction there is depending on the layer-to-layer increment as well as the in-plane rastering a certain roughness in the range of this increment. In general, a finer rastering and design discretization allows for a smoother surface while it directly impacts the total writing effort and time. E.g., the writing time would scale linearly in each direction, and thus an increase of overlap from zero to $50 \%$ of adjacent layers in only one direction would double the exposure time, and an overlap of $90 \%$ would multiply it by 10 . Thus, the method supports fast and large area preparation of original structures with rather coarse discretization that can be later replicated with methods such as nanoimprint lithography and post-processed using thermal reflow to save overall process time.

TASTE involves selectively changing the material properties of the part of the sample that needs modification, i.e., locally confined in lateral or vertical direction. It works particularly well with thermoplastic polymers such as PMMA. By use of high-energy exposure, the polymer is modified at a defined depth of the surface by chain scission that can be followed by molecular weight dependent reflow at elevated temperatures. In case of EBL, the high penetration depth of electrons with several $\mathrm{kV}$ typically exposes the entire film, which for polymethyl methacrylate (PMMA) is up to a few $\mu \mathrm{m}$. Therefore, TASTE can be confined laterally in a spincoated PMMA film serving as a resist or a surface topography material for molding. For surface smoothening of microlenses only the modification of a skin layer is required, which should be in the range of the height of the surface undulations but not exceeding the size of structural details in the micro-elements to be smoothened. For the microlenses made by 2PP-DLW the roughness was in the range of $100 \mathrm{~nm}$ and the smallest structural details around $1 \mu \mathrm{m}$. The 100 to $400 \mathrm{~nm}$ penetration depth we have found for $172 \mathrm{~nm}$ UV exposure are an ideal fit for this application. A lens design with a test micro-lens array $(3 \times 3)$ with concave lenses $50 \mu \mathrm{m} \times 50 \mu \mathrm{m}$ (each) and a height of $50 \mu \mathrm{m}$ from tips at the four corners to the central sag was chosen.

This simple structure would have taken $50 \mathrm{~min}$ of writing time when choosing a $50 \mathrm{~nm}$ slicing for smooth surfaces. Instead of the increase of overlap (small slicing increment), we chose a post-processing method which allows writing the micro-optical lenses with high speed, but large increments, and added a surface smoothening ("polishing") process after the writing (Figure 2). Since the process needs to be based on non-contact and should not involve a coating step, it requires a surface selective modification of the polymer in a depth that is comparable to the surface roughness we want to smoothen out. Such surface selective modification is possible by exposure of the polymer with intense radiation or high energy beams of electrons and ions (see Figure 2). Similarly to the process established for grayscale electron beam lithography structures, we have established a variant of the TASTE process for reduction of the molecular weight of the polymer and thus the glass transition temperature with a high degree of localized confinement [10]. 
The available methods for a vertically localized modification do not only differ in penetration depth, but due to their ways of interaction with the polymer surface also in surface ablation. For the purpose of surface smoothening, vacuum UV-light with $172 \mathrm{~nm}$ wavelength provided the best capabilities. It can be operated in ambient, and large area lamps enabling homogeneous irradiation (e.g., from Hamamatsu) are available. More information can be found in Ref. [24].

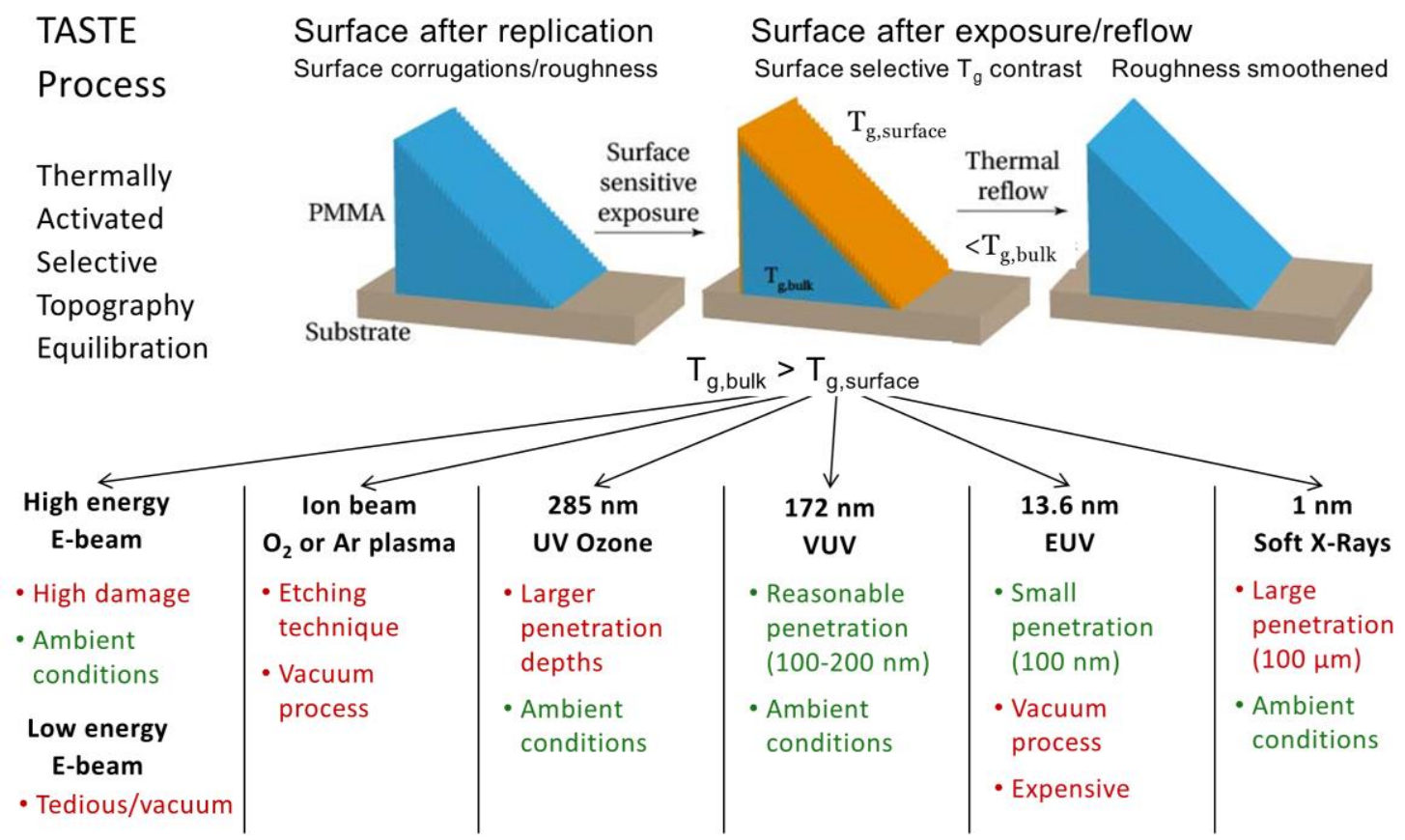

Figure 2. Different process possibilities for generating surface sensitive damage which can be used for thermally activated selective topography equilibration (TASTE): Exposure with low energy e-beam, $172 \mathrm{~nm}$ VUV and $13.6 \mathrm{~nm}$ EUV are known to enable damage in a skin layer of $100 \mathrm{~nm}$ only, with low ablation and roughening as it is the case for plasma treatment. VUV is available in commercial tools for large area exposure in ambient.

\section{PROCESS ROUTE FOR GENERATION OF REPLICATED MICROLENSES}

Since the TASTE process is best explored with linear thermoplastic polymers such as PMMA, but did not yet show good results with negative resists, we chose two replication steps that allowed transferring the lenses from the crosslinked proprietary material optimized for 2PP-DLW (IpDip and IpS from NanoScribe GmbH) to PMMA (Figure 3). For this, the lens structures were written with the NanoScribe Photonic GT in IpDip with high scan speeds provided by the galvanic scanner $(20 \mathrm{~mm} / \mathrm{sec}$ ) and low pulse intensity (around $1 \mathrm{~nJ}$ ), using a $500 \mathrm{~nm}$ slicing distance between adjacent layers. This resulted in a roughness of about $100 \mathrm{~nm}$. For comparison, the same structures were exposed in IpS, a resist optimized for higher throughput and lower surface roughness, with different slicing distances. The process chain for the upscaling of lens fabrication involves a further copying of lens structures by molding techniques, undercuts or "open" structures cannot be allowed. We therefore choose for the 2PP-DLW a method of bulk filling instead of writing a scaffold with reduced overlap, resulting in hollow structures, which would be prone to collapse and excessive shrinkage. In case of the concave lenses used here, core-shell writing strategies are less favorable as for convex lenses, where a thin skin could be written around the thick central core in which a quantity of liquid and non-polymerized resist could be contained and crosslinked by a flood exposure. For the writing of solid structures, the writing time is linearly dependent on the overlap between adjacent layers.

For the laser power of about $65 \%$ used for MLA manufacturing in this paper, the voxel size with a 63x NA 1.4 objective for the IpDip is typically about $260 \times 260 \times 1180 \mathrm{~nm}^{3}$ while for the IpS it is enlarged to $290 \times 290 \times 1100 \mathrm{~nm}^{3}$. For the $25 \mathrm{x}$ NA 0.8 objective the voxel size is about $300 \times 300 \times 3380 \mathrm{~nm}^{3}$ for the IpDip and about $380 \times 380 \times 3960 \mathrm{~nm}^{3}$ for the IpS, including the blurring due to the chemical nature of the resist. In a previous publication [8], on which this paper is based, 
we have anticipated smaller values $\left(200 \times 200 \times 600 \mathrm{~nm}^{3}\right.$ for IpDip and $63 \mathrm{x}$, and $300 \times 300 \times 900 \mathrm{~nm}^{3}$ for IpS and $\left.25 \mathrm{x}\right)$, which have now been improved by measurement [9]. Therefore, while the lateral extension of the voxels is quite small, their vertical extension is large and the height-width ratio ranges from 3.3 from $63 \mathrm{x}$ in IpS to 12 for $25 \mathrm{x}$ in IpDip. Thus, for the $500 \mathrm{~nm}$ slicing the vertical overlap is more than 50\% (IpS, 63x objective) and increases to almost 99\% (IpS, 25x objective) for $50 \mathrm{~nm}$ slicing. The writing time for a single lenslet in the $3 \times 3$ microlens array was $34 \mathrm{sec}$ for $500 \mathrm{~nm}$ slicing and $335 \mathrm{sec}$ for $50 \mathrm{~nm}$ slicing. For structures used for surface smoothening, the IpDip original was first copied into UV-PDMS (an alternative to Ormostamp, both from microresist technology $\mathrm{GmbH}$ ), which is flexible but at the same time hard enough that both the structures and the roughness can be replicated without loss, and then by thermal imprint into PMMA (see Figures 3 and 4). More details on the replication process can be found in Refs. [9,25-27].

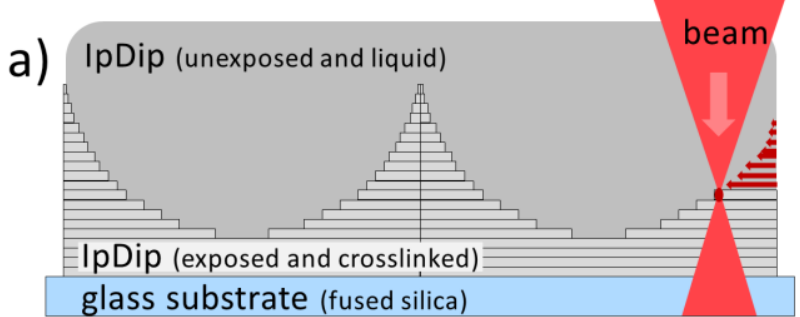

b)

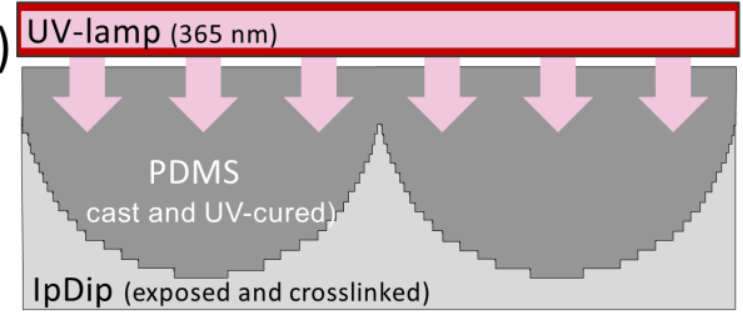

c)

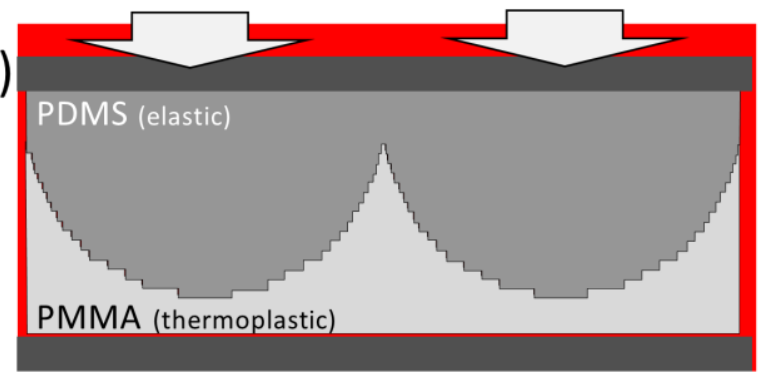

d)

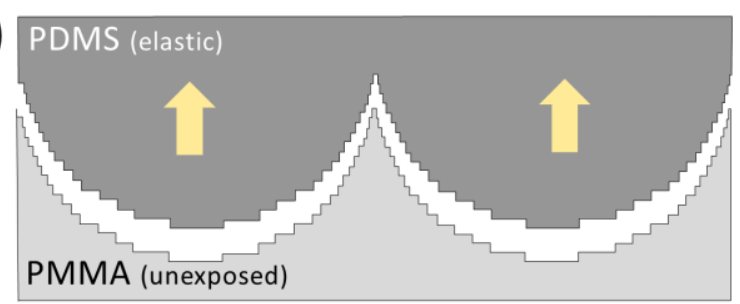

e)

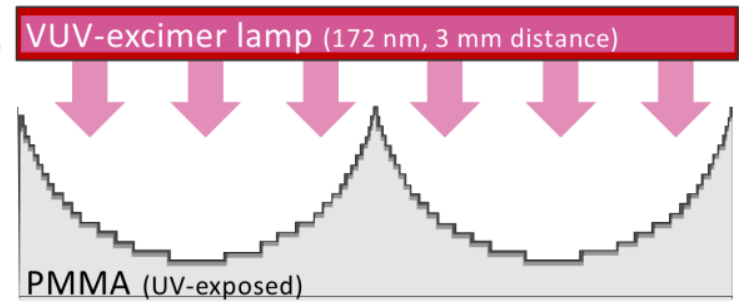

f)
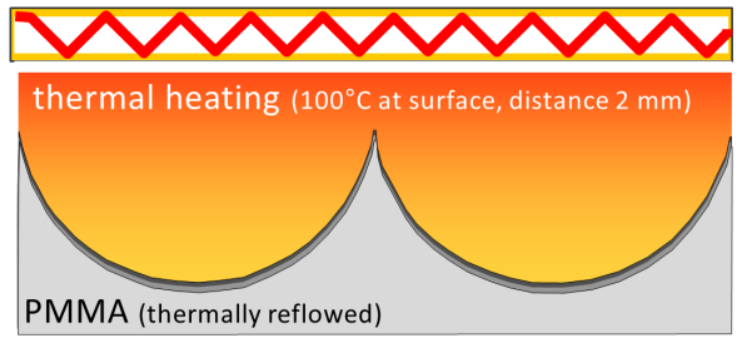

Figure 3. Process route for fabrication (origination), replication and polishing of micro-lenses. a) Two-photon direct write laser-lithography (2PP-DLW) of concave lenses in negative (IpDip) resist, b) Replication into UV-PDMS (convex lenses), c) thermal imprint into PMMA and demolding. d) Unexposed PMMA with roughness (same polarity as original concave structure), e) exposure with VUV-light, f) thermal reflow, which smoothens out surface roughness.
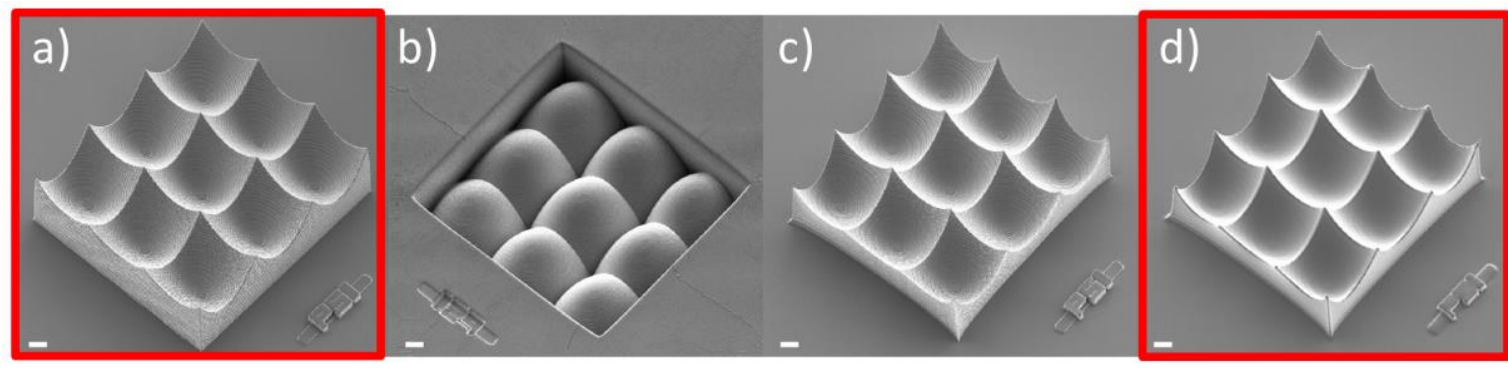

Figure 4. Process steps from a) original in IpDip (63x, slicing distance $500 \mathrm{~nm})$, b) replication by UV-NIL into UV-PDMS, c) thermal imprint into PMMA, d) surface smoothening by exposure and thermal reflow. Scale bar $10 \mu \mathrm{m}$. 


\section{RESULTS AND COMPARISON OF METHODS}

Figure 5 presents scanning electron micrographs (SEM) of a $3 \times 3$ microlens array manufactured by 2PP-DLW displaying the effect of variation of voxel size, writing strategy and post-processing on surface roughness. The $63 \mathrm{x}$ objective lens for the IpDip resist was optimized for highest (lateral) resolution, but also exhibits the most prominent roughness due to the small voxel size. The $25 \mathrm{x}$ lens was used for IpDip, too. This already significantly reduces the roughness, which is further reduced by the use of IpS, the resist developed for low surface roughness. This is achieved by the "intrinsic diffusion" of the resist, leading of a blurring of the exposed voxel, and the lower magnification as well as numerical aperture (NA) of the microscope objective. The slicing was kept constant, although the use of IpS in conjunction with the $25 \mathrm{x}$ lens would enable a larger slicing. Therefore, and since we choose a small writing scan field $(150 \mu \mathrm{m}$ in $\mathrm{x}$ and $\mathrm{y}$ direction), the in principal larger scanning field of the $25 \mathrm{x}$ lens in comparison to the $63 \mathrm{x}$ lens did not lead to higher throughput. All single lenslets in the $3 \times 3$ array (Figures $5 \mathrm{a}-\mathrm{c}$ ) were written with $34 \mathrm{sec}$ writing time.

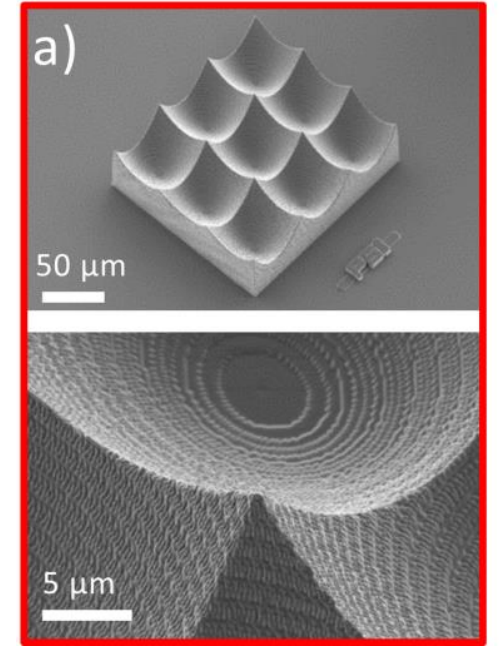

63x, IpDip, slicing $500 \mathrm{~nm}$
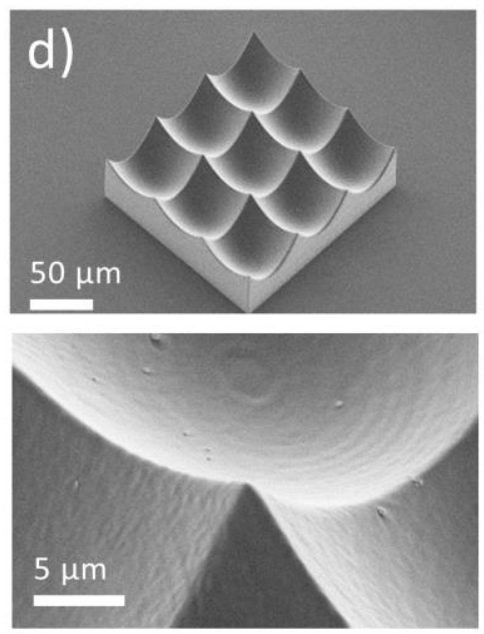

25x, IpS, slicing $300 \mathrm{~nm}$
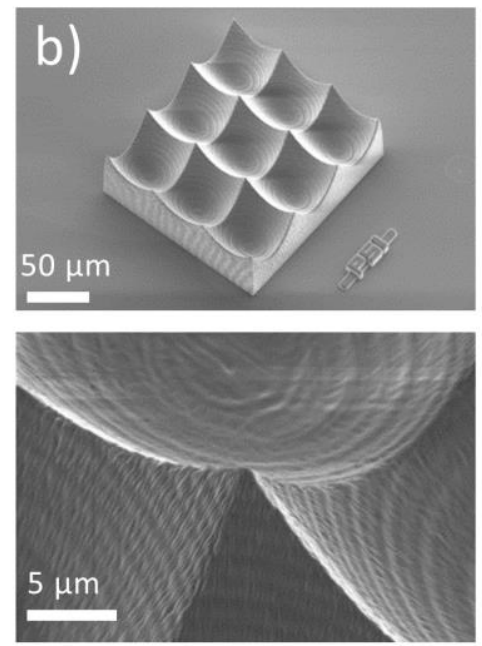

25x, IpDip, slicing $500 \mathrm{~nm}$
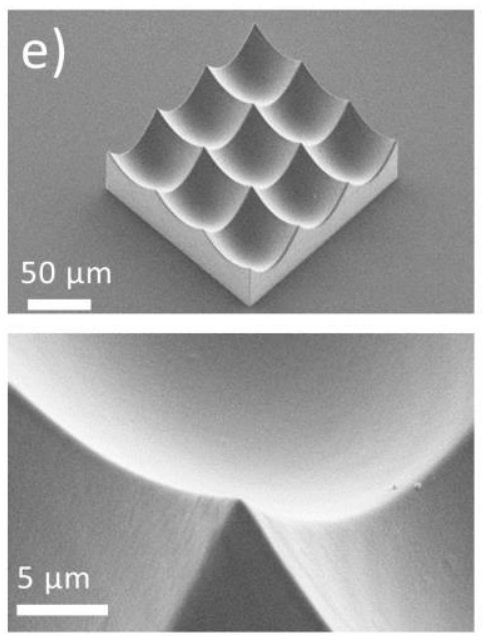

25x, IpS, slicing $50 \mathrm{~nm}$
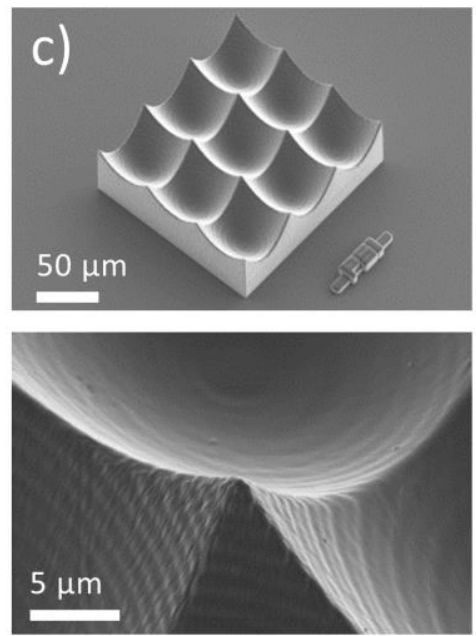

25x, IpS, slicing $500 \mathrm{~nm}$

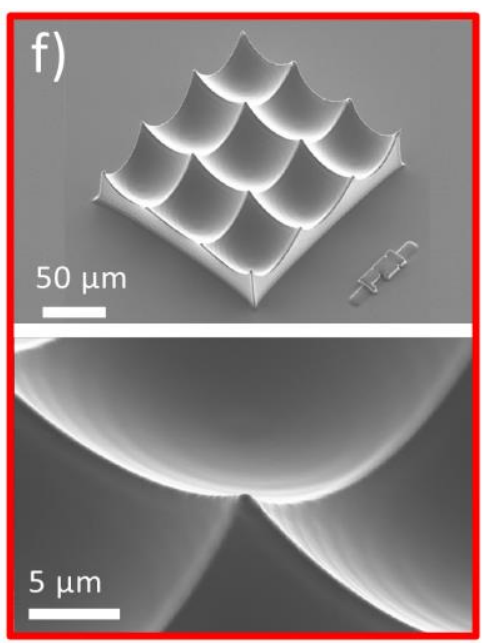

63x, IpS, slicing $500 \mathrm{~nm}$, reflow

Figure 5. SEM micrographs of microlens array manufactured by 2PP-DLW displaying the effect of variation of voxel size (resist with "intrinsic diffusion", magnification and NA of microscope objective), writing strategy (slicing) and postprocessing (TASTE) on surface roughness. Upper row: $3 \times 3$ lens array, lower row: magnification of intersection of 4 lenses. a-c) with different microscope lens and resists; slicing distance $500 \mathrm{~nm}$ constant (resulting in $34 \mathrm{sec}$ writing time per single lenslet): a) with $63 \mathrm{x}$ in IpDip, b) with $25 \mathrm{x}$ in IpDip, c) with $25 \mathrm{x}$ in IpS. d) and f) with different slicing distances both with 25x in IpS: d) $300 \mathrm{~nm}$ (in $56 \mathrm{sec}$ writing time per lenslet), e) $50 \mathrm{~nm}$ (in $335 \mathrm{sec}$ writing time per lenslet). f) For comparison with post-processing using selective thermal reflow with lenses in a) with $63 x$ in IpDip, indicated by colored frames. 
Further improvements in the surface roughness can be achieved by reduction of the slicing distance. Here, we show the reduction from $500 \mathrm{~nm}$ to $300 \mathrm{~nm}$, for which still some details of layering could be seen in the deep sags of the concave lenses (Figure $5 \mathrm{~d}$ ). For $50 \mathrm{~nm}$ slicing almost no roughness could be seen in the SEM micrograph (Figure 5 e). However, the micrograph displaying the lens, which was obtained using smoothening by selective thermal reflow still shows a superior surface finish (Figure $5 \mathrm{f}$ ). Although roughness values were not quantitatively analyzed using scanning force microcopy (SPM), both values displayed in Figure 5 e and Figure $5 \mathrm{f}$ are considered to be well below $10 \mathrm{~nm}$. This goes along with the results by other researchers in [3], where $10 \mathrm{~nm}$ roughness was obtained with $150 \mathrm{~nm}$ slicing with IpS in the solid writing mode. However, the writing time of the structure in IpS with $50 \mathrm{~nm}$ slicing is 10 times larger than that of the original structure in IpDip. Such differences in writing times will result in huge processing times, e.g., if lens arrays with a few millimeters of diameter need to be exposed. As an example, for a lens structure with $2.4 \mathrm{~mm}$ diameter, consisting of about 3'500 lenses with $50 \times 50 \mu \mathrm{m}^{2}$ footprint each, the exposure time is 48 hours, even for the $500 \mathrm{~nm}$ slicing. Considering the fact that the rough $500 \mathrm{~nm}$ slicing already involves a $58 \%$ overlap for the $63 \mathrm{x}$ objective (IpDip) and $87 \%$ for $25 \mathrm{x}$ objective (IpDip and IpS) in vertical direction, for which significant roughness has been observed, the needed overlap of $99 \%$ for optically smooth surfaces seems extreme.

Small differences, e.g., the tip at the intersection of the 4 lenses and the right slope of the surface smoothened structure in Figure $5 \mathrm{f}$ indicate some minor issues. Although high aspect ratio structures are prone to collapse, this was not observed for the surface smoothening. For the concave structures with highly varying slopes shown here, the surface smoothening seems to work over steps of up to $400 \mathrm{~nm}$, from the bottom with the shallow rings up to the sharp tips with the $<1 \mu \mathrm{m}$ radius. One can argue that surface reflow is a very unique equilibration process which allows not only a reduction of surface roughness initiated by the crosslinking in enlarged blurred voxel, but also an energy minimization which is based on material displacement from elevated areas (the center of the layers) into small depressions (the spaces between voxels or layers). However, one should not forget that the damage of the PMMA during exposure results in a surface skin with low molecular weight, which has to be taken into account for further process steps involving temperature treatment or aggressive solvents.

\section{CONCLUSION}

The aim of this focused review was to demonstrate the efforts necessary to achieve similar roughness with reducing the slicing distance as with smoothening by reflow. While several groups claim to achieve sub-10 $\mathrm{nm}$ roughness by the reduction of the slicing distance, it is questionable whether writing of high density trajectories to overcome the inherent roughness of 2PP-DLW is the right strategy to achieve surface qualities suitable for optical applications independent of the required geometry. Only if significant improvements in writing speed can be achieved, e.g., by developing resists with large process windows (low polymerization threshold and high damage threshold), or varying voxel sizes can be employed, such conservative approaches will enable to step into mass fabrication of micro-elements. They are more likely to be used for research or as originals for replication. Strategies to write shells on cores (skin modus) with smaller increment than the inner part (or even a scaffold) of the lens is the way to go if high throughput structures with resolution elements have to be directly written, or writing on prepatterned substrates which use mix- and match schemes and local focusing is required. As it has been shown here and in previous publications, the post-processing is a way to achieve low surface roughness from structures which were optimized for speed and not surface roughness. It has to be pointed out that, apart from small amounts of materials ablated during UV-exposure, the reflow is the only process which equilibrates surface undulations by local displacement of material and therefore creates a well-defined envelope. Due to the lack of availability of production machines for 2PP-DLW, throughput will remain the main issue for the fabrication of extended arrays of lenses. Scaling larger arrays and higher structures will critically enhance the writing time. Since in the future there will be both a need for original structures and such being used as originals for replication, different strategies need to be developed. The NIL process chain with its main elements origination, replication and pattern transfer (functionalization), is a good example to demonstrate this capability [28,29].

\section{ACKNOWLEDGEMENT}

This project was funded by the Swiss Nanoscience Institute (project A10.13 SurfFlow). The authors express their special thanks to K. Vogelsang (PSI) and M. Altana (Heptagon) for process support and measurements. The authors also thank M. Rossi (Heptagon) for the support of this project and the useful discussion. 


\section{REFERENCES}

[1] Zhou, X., Hou, Y., and Lin, J., "A review on the processing accuracy of two-photon polymerization," AIP Advances 5, 030701 (22 pp) (2015).

[2] Harnisch, E., Russew, M., Klein, J., König, N, Crailsheim, H. and Schmitt, R., "Optimization of hybrid polymer materials for $2 \mathrm{PP}$ and fabrication of individually designed hybrid microoptical elements thereof," Opt. Mat. Expr. 5(2), 456-461 (2015).

[3] Gissibl, T., Thiele, S., Herkommer, A., and Giessen, H., "Two-photon direct laser writing of ultracompact multi-lens objectives," Nature Photonics 10, 554-560 (2016).

[4] Popovic Z.D., Sprague, R.A., and Connell, G.A.N., "Technique for monolithic fabrication of microlens arrays," Appl. Opt. 27, 1281-4 (1988).

[5] Eckhardt Optics LLC, online, http://eckop.com/optical-scatter-2/optical-scattering-versus-surface-roughness/, accessed 2017-01-23.

[6] Bennett, H. E. and Porteus, J. O., "Relation between surface roughness and specular reflectance at normal incidence," J. Opt. Soc. of Am. 51, 123-129 (1961).

[7] Chidambaram, N., Kirchner, R. Fallica, R., Yu, L., Altana, M., and Schift, H., "Selective surface smoothening of polymer microlenses by depth confined softening," Adv. Mater. Technol. 1700018, (10 pp) (2017).

[8] Kirchner, R., Chidambaram, N., Altana, M., and Schift, H., "How post-processing by selective thermal reflow can reduce the roughness of 3D lithography in micro-optical lenses," Proc. SPIE. 10095, Laser 3D Manufacturing IV, 1009507 (9 pp) (February 24, 2017).

[9] Kirchner, R., Chidambaram, N., and Schift, H., "Thermal post-processing of selectively modified thermoplastic surfaces for ultra-smooth 3D-printed micro-optics," Optical Engineering, to be published (2017).

[10] Schleunitz, A. and Schift, H., "Fabrication of 3D nanoimprint stamps with continuous reliefs using dosemodulated electron beam lithography and thermal reflow," J. Micromech. Microeng. 20, 095002 (6pp) (2010).

[11] Schleunitz, A., Guzenko, V. A., Messerschmidt, M., Atasoy, H., Kirchner, R., and Schift, H., "Novel 3D microand nanofabrication method using thermally activated selective topography equilibration (TASTE) of polymers," Nano Convergence 1:7, (2014).

[12] Gale, M.T., Rossi, M., and Schuetz, H., "Fabrication of continuous-relief micro-optical elements by direct laser writing in photoresist, laser-assisted fabrication of thin films and microstructures," Proc. SPIE 2045, 54-62 (1994).

[13] Kirchner, R., Guzenko, V. A., Vartiainen, I.; Chidambaram, N. ,and Schift, H., "ZEP520A - A resist for electron-beam grayscale lithography and thermal reflow," Microelectron. Eng. 153, $71-76$ (2016).

[14] Kirchner, R., Guzenko, V. A., Rohn, M., Sonntag, E., Mühlberger, M., Bergmair, I., and Schift, H., "Bioinspired 3D funnel structures made by grayscale electron-beam patterning and selective topography equilibration," Microelectron. Eng. 141, 107-111 (2015).

[15] Stavis, S. M., Strychalski, E. A., and Gaitan, M., "Nanofluidic structures with complex three-dimensional surfaces," Nanotechnology 20, 165302 (7 pp) (2009).

[16] Keskinbora, K., Grévent, C., Hirscher, M., Weigand, M. and Schütz, G., "Single-Step 3D nanofabrication of kinoform optics via gray-scale focused ion beam lithography for efficient X-ray focusing," Adv. Opt. Mater. 3, 792-800 (2015).

[17] Maruo, S., Nakamura O., and Kawata S., "Three-dimensional microfabrication with two-photon-absorbed photopolymerization," Opt. Lett. 22(2), 132-134 (1997).

[18] Chidambaram, N., Kirchner, R., Altana, M., and Schift, H., "High fidelity 3D thermal nanoimprint with UV curable polydimethyl siloxane stamps," J. Vac. Sci. Technol. B 34(6), 06K401 (2016).

[19] Dietrich, P.-I., Reuter, I., Blaicher, M., Schneider, S., Billah, M. R., Hoose, T., Hofmann, A., Caer, C., Dangel, R., Offrein, B., Moehrle, M., Troppenz, U., Zander, M., Freude, W., and Koos, C., "Lenses for low-loss chip-tofiber and fiber-to-fiber coupling fabricated by 3D direct-write lithography," Conf. on Lasers and Electro-Optics (CLEO'16), San Jose (CA), USA, June 5 - 10 , paper SM1G.4. (2016).

[20] Takada, K., Sun, H.B., and Kawata, S., "Improved spatial resolution and surface roughness in photopolymerization-based laser nanowriting," Applied Physics Letters 86, 071122, (2005).

[21] Stuart, B.C., Feit, M.D., Herman, S., Rubenchik, A.M., Shore, B.W., and Perry, M.D., "Nanosecond-tofemtosecond laser-induced breakdown in dielectrics," Physical Review B 53(4), 1749 (1996).

[22] Guo, R., Xiao, S., Zhai, X., Li, J., Xia, A., and Huang, W., "Micro lens fabrication by means of femtosecond two photon photopolymerization," Optics Express 14, 810-816 (2006). 
[23] Wu, D., Wu, S.Z., Niu, L.G., Chen, Q.D., Wang, R., Song, J. F., Fang, H.H., and Sun, H.B., "High numerical aperture microlens arrays of close packing," Applied Physics Letters 97, 031109 (2010).

[24] Kirchner, R., Hoekstra, R., Chidambaram, N., and Schift, H., "Depth-profiling of vertical material contrast after VUV exposure for contact-free polishing of 3D polymer micro-optics," Proc. SPIE, EMLC (8 pp) (2017).

[25] Schift, H., Chidambaram, N., Altana, M., and Kirchner, R., "How post-processing by selective thermal reflow can reduce the roughness of 3D lithography in micro-optical lenses," Proc. SPIE. 10144, Alternative Lithographic Techniques, 101440B (7 pp) (2017).

[26] Schift, H., Spreu, C., Saidani, M., Bednarzik, M., Gobrecht, J., Klukowska, A., Reuther, F., Gruetzner, G. and Solak, H.H., "Transparent hybrid polymer stamp copies with sub-50 nm resolution for thermal and UVnanoimprint lithography," J. Vac. Sci. Technol. B 27(6), 2846-2849 (2009).

[27] Schift, H., "Nanoimprint lithography: an old story in modern times? A review," J. Vac. Sci. Technol. B 26(2), 458-480 (2008).

[28] Schift, H., Urwyler, P., Kristiansen, P.M., and Gobrecht, J., "Nanoimprint lithography process chains for the fabrication of micro- and nanodevices," J. Micro/Nanolith. MEMS MOEMS 13(3), 031303 (2014).

[29] Schift, H., "Nanoimprint lithography, 2D or not 2D? A review," Appl. Phys. A 21(2), 415-435 (2015). 